\title{
SOBRE EL MINIMALISMO ANTI-CONFLICTIVISTA Y LIBERAL: UN ANÁLISIS DESDE LA PERSPECTIVA DE LA INTEREST THEORYY YEL ENFOQUE DINÁMICO
}

\author{
ANTI-CONFLICTUALIST AND LIBERAL MINIMALISM OF RIGHTS: \\ AN ANALYSIS FROM THE PERSPECTIVE \\ OF INTEREST THEORY AND THE DYNAMIC APPROACH
}

\author{
Michele ZeZZA* \\ Universidade de São Paulo (USP) \\ Brasil
}

Fecha de recepción: 11-1-20

Fecha de aceptación: 15-9-20

Resumen: El artículo describe dos enfoques minimalistas en materia de reconocimiento y protección de los derechos: las variantes anti-conflictivista y liberal. En ello, se reconstruyen sus fundamentos histórico-culturales y se destacan los principales elementos de su insostenibilidad teórica. Para este fin, se utilizan algunas herramientas teóricas derivadas de la combinación de la interest theory con un enfoque dinámico.

Abstract: The article describes two different minimalist approaches in the field of recognition and protection of rights: the anti-conflictual and the liberal variants. It explores their historical-cultural assumptions and highlights their main elements of their unreasonableness. To this end, we use some theoretical tools deriving from the combination of interest theory with a dynamic approach.

Palabras clave: minimalismo anti-conflictivista, minimalismo liberal, interest theory, enfoque dinámico

Keywords: anti-conflictualist minimalism, liberal minimalism, interest theory, dynamic approach

* El presente trabajo ha sido financiado por la Fundação de Amparo à Pesquisa do Estado de São Paulo (FAPESP), con el siguiente número de proceso: 2017/24035-9.

ISSN: 1133-0937

DOI: https://doi.org/10.20318/dyl.2021.6109
DERECHOS Y LIBERTADES

Número 45, Época II, junio 2021, pp. 303-326 


\section{EL ENFOQUE MINIMALISTA Y SUS RAÍCES HISTÓRICO- CULTURALES}

Incluso desde un análisis superficial de su desarrollo histórico, es inevitable notar cómo, a partir del constitucionalismo de la segunda posguerra, la noción de derecho subjetivo ha registrado un significativo proceso de transformación que ha afectado tanto el plano de las reivindicaciones como el de las adscripciones. Paralelamente a la multiplicación de las exigencias sociales de los ciudadanos, incluso la cantidad de los titulares de los derechos (individuos, comunidades, animales, plantas, objetos inanimados, generaciones futuras, etc.) ha aumentado considerablemente, hasta el punto de incluir en ciertas ocasiones nuevos ${ }^{1}$ sujetos jurídicos e intereses colectivos, así como peculiares herramientas de reconocimiento y protección jurídica.

En comparación con las fases anteriores de la evolución de los derechos referidas a las tradicionales libertades individuales y a los derechos sociales, económicos y culturales- la actual estrategia reivindicativa de los derechos exhibe algunos rasgos indudablemente peculiares. Sólo para poner algunos ejemplos: asuntos como las garantías frente a los riesgos de manipulación genética, los derechos del embrión o el derecho al aborto, el derecho al desarrollo o a una suficiente calidad de vida, han adquirido, y continúan adquiriendo, una relevancia innegable en el marco de las exigencias individuales y colectivas que caracterizan el constitucionalismo contemporáneo.

Nos encontramos frente a un conjunto heterogéneo de situaciones subjetivas, cuya inclusión en las clasificaciones tradicionales es a menudo difícil de alcanzar. En este sentido, dentro de las actuales sociedades globalizadas y multiculturales, las reivindicaciones que suelen ser incluidas en el marco de los derechos de cuarta generación (derechos colectivos y derechos de solidaridad) componen un catálogo abierto, dinámico y flexible de derechos que raramente son justiciables, o incluso no reconocidos por el derecho positivo. Existe una tendencia creciente, dentro del debate filosófico-jurídico sobre los derechos más recientes, a extender la noción de titularidad a sujetos no-humanos que, en sentido estricto, carecen de una plena capacidad jurídica y, en algunos casos, incluso de la capacidad de expresar su propia voluntad. De esta manera, se produce un proceso de fragmentación del originario sujeto

\footnotetext{
1 Cabe señalar que aquellos derechos que, en el debate teórico, suelen ser llamados "nuevos derechos", en realidad, en la mayoría de los casos son reformulaciones (nuevas interpretaciones o nuevas aplicaciones) de derechos ya existentes.
} 
de los derechos, tradicionalmente identificado con un individuo dotado de características como la autonomía, la racionalidad y la intangibilidad.

La configuración especial que el reconocimiento y la titularidad de los derechos fundamentales ha adquirido en los Estados constitucionales en las últimas décadas ha tenido como consecuencia el nacimiento y el desarrollo de un conjunto de propuestas normativas que conciben el problema de la expansión anómica de los derechos como uno de los principales factores de crisis del constitucionalismo contemporáneo. Estos planteamientos suelen detectar una relación de proporcionalidad inversa entre la tendencia -efectivamente difundida en el lenguaje jurídico-político contemporáneo- a representar innumerables reivindicaciones en términos de derechos y su efectiva capacidad de satisfacer los intereses y las necesidades de las personas: al reconocimiento cada vez más amplio de la titularidad de derechos y de instrumentos de tutela se corresponde, por tanto, una disminución progresiva de su eficacia ${ }^{2}$.

En este sentido, observa Roberto Bin:

la desafinación es evidente: la venta de títulos nobiliarios es el preludio de la pérdida de su relevancia social, y lo mismo se aplica al estatus constitucional de los "derechos". Conceder el estatus de derecho constitucionalmente reconocido a intereses que proceden de la puerta pequeña de una interpretación audaz hace muy poco creible el intento de recurrir a este estatus para desvitalizar otros intereses antagónicos, desprovistos de un título nobiliario ${ }^{3}$.

Un paso adicional, en relación con este discurso, consiste en sostener que, para evitar el debilitamiento de los bienes moral y jurídicamente relevantes, sería conveniente limitar su reconocimiento y protección jurídica a aquellos intereses más vinculados a las tradicionales libertades negativas ${ }^{4}$, entendidos como perfectamente cerrados en sí mismos, de fácil o inmediata aplicación, intrínsecamente aconflictivos y gratuitos (o baratos) ${ }^{5}$. Se propo-

2 D. ZOLO, “Libertà, proprietà ed eguaglianza nella teoria dei 'diritti fondamentali' . A proposito di un saggio di Luigi Ferrajoli”, Teoria politica, núm. 15, vol. 1, 1999, pp. 16-18, habla de una "ley de la efectividad decreciente de las garantías de los derechos".

3 R. BIN, “Diritti e fraintendimenti", Ragion Pratica, núm. 14, 2000, pp. 23-24, traducción mía. Es conveniente precisar que el autor no defiende un enfoque minimalista en materia de reconocimiento y protección de los derechos.

4 Para la noción de libertad negativa, la referencia principal es I. BERLIN, Two concepts of liberty: an inaugural lecture delivered before the University of Oxford on 31 October 1958, Oxford Clarendon Press, Oxford, 1958.

5 Versiones emblemáticas de esta perspectiva pueden ser halladas en M. CRANSTON, "Human Rights, Real and Supposed", en D.D. RAPHAEL (ed.), Political Theory and Rights 
ne, así, una redefinición del catálogo de los derechos (y de sus garantías) en una dirección marcadamente deflacionista o, cuando menos, orientada a deconstruir el principio de la indivisibilidad de los derechos. En esta perspectiva, se considera la restricción de la cantidad de los intereses moral y constitucionalmente legítimos como una forma de evitar que los derechos "reales" sean debilitados ${ }^{6}$.

El proceso de especificación de los bienes y titulares, la difusión cada vez más amplia de discursos, reclamaciones y demandas de derechos puede producir a menudo un incremento de las ocasiones de conflicto entre los mismos derechos y constituir un obstáculo objetivo para su protección. En las reflexiones siguientes centraremos la atención sobre dos modelos de minimalismo particularmente relevantes dentro del debate filosófico-político y filosófico-jurídico actual: 1) un enfoque que podría definirse como "anti-conflictivista", cuyo propósito principal es resolver o limitar los problemas de incompatibilidad entre derechos; y 2) un enfoque "liberal", que apunta en cambio a restringir el catálogo de los derechos a las libertades fundamentales clásicas.

\section{EL MINIMALISMO ANTI-CONFLICTIVISTA}

En términos generales, el enfoque conflictivista tiende a considerar el problema de la incompatibilidad entre derechos ${ }^{7}$ como una relación antinó-

of Man, Macmillan, London, 1967; M. IGNATIEFF, Human Rights as Politics and Idolatry, Princeton University Press, Princeton, Oxford, 2001; O. O'NEILL, "The Dark Side of Human Rights", International Affairs, núm. 81, vol. 2, 2005, pp. 427-439; R. NOZICK, Anarchy, State, and Utopia [1974], Wiley-Blackwell, 2011; T. NAGEL, Mortal questions [1979], Cambridge University Press, Cambridge, 2013.

6 Un enfoque parcialmente parecido es el defendido por John Rawls en The Law of Peoples, en el que el autor extiende las nociones de 'justicia como equidad' y de 'liberalismo político', originalmente diseñadas para sociedades nacionales particulares, en una perspectiva internacional, proponiendo de hecho, a nivel global, ideas análogas a las que los críticos libertarios de su teoría habían elaborado en referencia a la etapa inicial de su teoría. La cantidad de los derechos humanos y la calidad de su protección son limitados, ya que en una teoría de la justicia internacional representan una restricción a la soberanía y están esencialmente vinculados al respeto del principio de autodeterminación. Por tanto, los derechos humanos son concebidos como un umbral mínimo a alcanzar para formar parte de la Sociedad de los pueblos (v. J. RAWLS, The Law of Peoples with "The Idea of Public Reason Revisited", Harvard College, Cambridge, Massachusetts, 1999; al respecto, véase también la crítica de J. GRIFFIN, On Human Rights, Oxford University Press, Oxford, 2008, pp. 22-27).

7 Para una introducción al tema del conflicto entre derechos: R. ALEXY, Teoría de los derechos fundamentales [1985], tr. esp., Centro de Estudios Constitucionales, Madrid, 2007, pp. 
mica, de "incoherencia lógica" ("inconsistency") ${ }^{8}$, entre normas (generalmente principios) que reconocen derechos fundamentales, imputables a sujetos que se encuentran en una posición antagónica o incluso al mismo sujeto. En esta situación, los deberes involucrados no resultan "composibles" o aplicables de forma consistente, como explica Hillel Steiner:

[l] incomposibilidad entre derechos puede asumir cualquiera de estas dos dimensiones: puede haber incompatibilidad entre el ejercicio de diferentes tipos de derechos por parte de diferentes personas; o puede existir entre diferentes personas que ejercen el mismo tipo de derecho. Mi ejercicio de mi derecho a la libertad de expresión puede interferir con el ejercicio de su derecho a la privacidad; o mi ejercicio de mi derecho a la libertad de expresión puede interferir con su ejercicio de su derecho a la libertad de expresión ${ }^{9}$.

Se trata por tanto de un problema de aplicación de normas (atributivas de derechos fundamentales) que ocurre dentro de los sistemas jurídicos cada vez que no sea posible cumplir al mismo tiempo con dos exigencias reconocidas en ellos: la implementación de un derecho que pertenece a un individuo $x$ requiere, por razones conceptuales o fácticas (es decir, in abstracto o in concreto), el incumplimiento de otro derecho (que también puede representar otra instancia del mismo derecho) de un individuo $y$.

En oposición a este discurso, diferentes planteamientos normativos niegan que, en abstracto, puedan ocurrir casos genuinos de colisión entre derechos, o afirman que, en caso de que se produzcan en la fase de aplicación, pueden ser solucionados estableciendo cuál derecho debe prevalecer ${ }^{10}$.

70-78; S. BESSON, The Morality of Conflict. Reasonable Disagreement and the Law, Hart, Oxford, 2005, pp. 430-436; J. WALDRON, Rights in Conflict [1989], en Liberal Rights: collected papers 1981-91, Cambridge University Press, Cambridge, 1993; F.M. KAMM, "Conflicts of Rights: Typology, Methodology and Non-Consequentialism”, Legal Theory, núm. 7, 2001, pp. 239-255.

8 Para una distinción entre la noción de "consistency" (compatibilidad lógica) y la de "coherence" (congruencia substancial) entre normas, véase N. MACCORMICK, Coherence in Legal Justification, en A. PECZENIK et al. (eds.), Theory of Legal Science, Reidel, Dordrecht, 1984, pp. 235-251.

9 H. STEINER, "The Structure of a set of Compossible Rights", Journal of Philosophy, núm. 74, vol. 12, 1977, p. 768, traducción mía. Sobre este tema, véanse también S. BESSON, The Morality of Conflict, cit.; J. GRIFFIN, On Human Rights, cit., pp. 57-82.

10 Entre las teorías normativas, ejemplos significativos pueden ser encontrados en: J. HABERMAS, Facticidad y validez: sobre el derecho y el estado democrático de derecho en términos de teoría del discurso [1992], tr. esp., Trotta, Madrid, 1998; I. DE OTTO, "La regulación del ejercicio de los derechos y libertades. La garantía de su contenido esencial en el artículo 53.1 de la Constitución", en Id., L. MARTÍN-RETORTILLO (a cargo de), Derechos fundamentales y

ISSN: 1133-0937

DOI: https://doi.org/10.20318/dyl.2021.6109
DERECHOS Y LIBERTADES

Número 45, Época II, junio 2021, pp. 303-326 
Según esta perspectiva, el error que habitualmente se comete a la hora de identificar un conflicto entre derechos radica en no precisar el antecedente de las normas que atribuyen derechos fundamentales. En una situación de presunto conflicto, o uno de los dos derechos involucrados no es un auténtico derecho, o se están ignorando los perímetros exactos de uno (o ambos) de ellos ${ }^{11}$. Los derechos entrarían en conflicto sólo en su formulación genérica, pero luego resultarían susceptibles de especificación, tras haber identificado una tercera regla cuya formulación haría desaparecer la apariencia de conflicto.

Dentro del debate filosófico-político, un ejemplo esclarecedor de esta primera forma de minimalismo es la argumentación desarrollada por Robert Nozick para neutralizar las hipótesis de colisión de derechos dentro de una teoría (mínima) del Estado. Esta reconstrucción forma parte de un proyecto más amplio de transformación utópica de la sociedad: en este marco, por tanto, hay que colocar también la intención de restringir la categoría de intereses que pueden aspirar a la calificación de "derechos" exclusivamente a las libertades negativas clásicas de abstención. Estos espacios de libertad pueden operar entonces como "vínculos colaterales": como ciertos límites absolutos y negativos a la conducta de las personas ${ }^{12}$. Aunque el autor no explicite claramente su contenido, de un análisis global de Anarquía, Estado y Utopía emerge la inspiración fuertemente individualista de una teoría centrada en el principio de la inviolabilidad de la persona.

Constitución, Tecnos, Madrid, 1988; J. JIMÉNEZ CAMPO, Derechos fundamentales. Concepto y garantías, Trotta, Madrid, 1999; A.L. MARTÍNEZ PUJALTE, La garantía del contenido esencial de los derechos fundamentales, Centro de Estudios Constitucionales, Madrid, 1997; J.J. MORESO, "Conflictos entre principios constitucionales", en Id. (a cargo de), Neoconstitucionalismo(s), Trotta, Madrid, 2003; Id., Dos concepciones de la aplicación de las normas de derechos fundamentales, en J. BETEGÓN et al. (a cargo de), Constitución y derechos fundamentales, Centro de Estudios Políticos y Constitucionales, Madrid, 2004, pp. 473-493; A. OLLERO TASSARA, "La ponderación delimitadora de los derechos humanos: libertad informativa e intimidad personal", Pensamiento y Cultura, núm. 3, 2000, pp. 157-166; J. CIANCIARDO, El conflictivismo en los derechos fundamentales, Eunsa, Pamplona, 2000; P. SERNA BERMÚDEZ, F.M. TOLLER, La interpretación constitucional de los derechos fundamentales: una alternativa a los conflictos de derechos, La Ley, Buenos Aires, 2000.

11 V. T. SMITH, "Rights Conflicts. The Undoing of Rights", Journal of Social Philosophy, núm. 26, 1995, en particular pp. 150-151.

12 V. R. NOZICK, Anarchy, State, and Utopia, cit., pp. 47-48. Esta concepción de los vínculos colaterales refleja el principio kantiano subyacente de los individuos como fines en sí mismos, sobre cuya base los derechos de un individuo no pueden ser vulnerados para evitar violaciones de derechos de otros sujetos. 
Para entender el significado de este discurso es preciso tener como punto de partida el problema del origen de la titularidad de los bienes. Al respecto, Nozick elabora una teoría pura del título válido ("entitlement theory"), es decir, orientada a determinar si las personas tienen efectivamente derecho a lo que poseen, que se estructura en torno a tres presupuestos esenciales: 1) un principio de justicia en la adquisición, que trata de la toma de posesión inicial de los bienes (cómo las personas llegan a poseer bienes naturales y sin dueño, qué tipos de objetos se pueden tener, etc.); 2) un principio de justicia en la transferencia, que explica cómo una persona puede adquirir propiedades de otra, incluyendo el intercambio voluntario y las donaciones; 3 ) un principio de rectificación de la injusticia, que explica cómo lidiar con las propiedades adquiridas o transferidas injustamente, cómo y en qué medida indemnizar a las víctimas, cómo tratar las transgresiones del pasado o las injusticias cometidas por un gobierno, y así sucesivamente ${ }^{13}$. En relación con este último punto, en teoría, este principio exige la reparación de las posibles injusticias cometidas a través de adquisiciones y transferencias previas: puede implicar, por tanto, incluso una compensación sustancial en beneficio de quienes han sido ilegítimamente perjudicados. En algunos contextos, aunque por un tiempo limitado, será necesario un fuerte intervencionismo estatal para mejorar la situación de los sujetos y grupos sociales más penalizados. Sin embargo, cabe señalar que Nozick no proporciona ninguna explicación sistemática en lo que respecta a cuándo y en qué medida los abusos de apropiación -la adquisición de propiedad en condiciones de escasez para el resto de la comunidad-deben ser modificados o sancionados ${ }^{14}$.

Esta perspectiva, encaminada a resaltar las condiciones de legitimidad de la apropiación privada de los recursos naturales, se funda abiertamente en la teoría del trabajo y la propiedad desarrollada por John Locke en el Segundo tratado sobre el gobierno civil, que en particular en el par. 27 ofrece una reconstrucción lógica del proceso de apropiación de los bienes naturales por parte de los individuos. En este sentido, Nozick retoma la teoría clásica del estado de naturaleza, entendida como aquella condición en la que cada persona puede alcanzar un cierto nivel de bienestar de acuerdo con sus propias habilidades. Tal bienestar, aunque distribuido de manera desigual, puede

$13 \quad$ Ibid., pp. 152-153.

14 Una crítica al individualismo nozickiano, que destaca su indiferencia respecto a las exigencias económico-sociales del bienestar colectivo, se puede encontrar en H.L.A. HART, “Between Utility and Rights”, Columbia Law Review, núm. 79, vol. 5, 1979, pp. 828-846. 
justificarse mediante lo que en la teoría del autor es la "cláusula lockiana" ("Lockean proviso") ${ }^{15}$, con arreglo a la cual la auto-pertenencia ("self-ownershi $p^{\prime \prime}$ ) proporciona a cada sujeto la libertad de agregar el resultado de su propio trabajo a los recursos naturales, trasformando la propiedad común en propiedad privada. En la reinterpretación nozickiana, esta cláusula limitadora implica que, aunque toda adquisición reduzca paralelamente el espacio de disponibilidad para los demás individuos, esta posesión es aceptable hasta que la condición de algún individuo no se vuelva peor de lo que habría sido sin alguna propiedad privada.

Este argumento lockiano, conocido como principio del labour-mixing, ha sido criticado por el proprio Nozick, quien pone en tela de juicio la idea de que añadir algo de propio a un objeto que anteriormente no pertenecía a nadie pueda fundar, ipso facto, la propiedad sobre este último: de hecho, este principio no puede constituir la justificación última del derecho a la adquisición de los recursos naturales, que en esta reconstrucción se delega a la función desempeñada por la cláusula limitadora. En este sentido, se pregunta Nozick: “ ¿Por qué mezclar lo que poseo con lo que no poseo es una forma de perder lo que tengo en lugar de una forma de ganar lo que no? Si poseo una lata de jugo de tomate y la derramo en el mar para que sus moléculas (radiactivas, para que pueda verificarlo) se mezclen uniformemente en todo el mar, ¿acaso llego a ser dueño del mar, o he disipado neciamente mi jugo de tomate?"16. Ahora bien, de acuerdo con una interpretación "débil" de la

15 Cfr. R. NOZICK, Anarchy, State, and Utopia, cit., pp. 175-182. En cierta medida, este enfoque puede ser relacionado con la teoría, elaborada por R. POSNER (Economic Analysis of law, Little Brown \& Co., Boston, 1977, pp. 36 ss.), de los derechos de apropiación. Sobre la cláusula lockiana, v. J. WOLFF, Robert Nozick: Property, Justice, and the Minimal State, Stanford University Press, Stanford, 1991; D. AI-THU, “Libéralisme et justice sociale: la clause lockéenne des droits de propriété", Revue française d'économie, núm. 10, vol. 4, 1995, pp. 205-238; J. WALDRON, "Nozick and Locke: Filling the space of rights", Social Philosophy and Policy, núm. 22, 1, 2005, pp. 81-110. Sobre la teoría lockiana: C.B. MACPHERSON, The Political Theory of Possessive Individualism: Hobbes to Locke, Oxford University Press, Oxford, 1962; J. TULLY, A Discourse on Property: John Locke and His Adversaries, Cambridge University Press, Cambridge, 1980; M. KRAMER, John Locke and the Origins of Private Property: Philosophical Explorations of Individualism, Cambridge University Press, Cambridge, 1997.

16 R. NOZICK, Anarchy, State, and Utopia, cit., pp. 174-175, traducción mía. Del mismo modo, Jeremy Waldron imputa a Locke un "category mistake" (más exactamente, un error semántico consistente en atribuir indebidamente una propiedad a un objeto), ya que el trabajo constituye una actividad que no puede ser identificada con las entidades físicas (v. J. WALDRON, "Two Worries About Mixing One's Labour", The Philosophical Quarterly, núm. 33, 130, 1983, pp. 37-44). 
cláusula limitadora, no reconducible al texto lockiano en su literalidad ${ }^{17}$, siempre deberá quedar para los demás la posibilidad como en la situación antecedente y sin pérdidas significativas en términos de bienestar. "La apropiación privada y unilateral de un recurso $\mathrm{R}$ es, según Nozick, legitima si no empeora la posición de los demás, que ya no tienen la libertad de hacer uso de $\mathrm{R}$, en lo referente a la situación inicial en que no existía propiedad privada sobre $R^{\prime \prime}{ }^{18}$. Toda persona -alega- debería preocuparse únicamente de respetar aquellos vínculos que hacen posible el ejercicio de los derechos de los demás: la posible vulneración del derecho del sujeto $A$ no exime el sujeto $B$ del deber de respetarlos. En resumidas cuentas: los títulos sobre los recursos colectivos -que Nozick concibe como sin propietario y no, como sostenía Locke, en condición de propiedad común- pueden considerarse legítimos si (a) se forman a través de la extensión a ellas de la propiedad que cada uno tiene sobre sí mismo y (b) se fundan en el respeto de la propiedad de sí $^{19}$.

Habida cuenta de lo que antecede, por tanto, los derechos se configuran como aquellos confines ("boundaries") que delimitan la legítima e inviolable esfera de acción de los individuos. Estas barreras no pueden ser superadas sin el consentimiento de las partes interesadas, por definición legitimadas a construirse libremente el propio futuro. Dentro de esta reconstrucción, los únicos derechos positivos admisibles son los que provienen de transacciones voluntarias entre personas (como los que surgen cuando se contratan determinados servicios esenciales).

En la base de esta propuesta normativa y deontológica se puede identificar la pretensión (objetivista) de definir el contenido de los derechos en función de un análisis abstracto de sus elementos teóricos: el problema de

17 El razonamiento lockiano puede ser sintetizado en los siguientes términos: “ $a$ ) toda persona $\mathrm{P}$ es propietaria de su trabajo $\mathrm{T} ; b$ ) trabajando en un recurso natural $\mathrm{R}$ común para todos, una persona mezcla $\mathrm{T}$ con R; entonces $c$ ) R contiene algo que pertenece a P; $d$ ) R es propiedad de $\mathrm{P}$; e) a condición de que la sobrevivencia de las otras personas no se ponga en peligro" (C. DEL BÒ, I diritti sulle cose: Teorie della giustizia e validità dei titoli, Carocci, Roma, 2008, p. 63, traducción mía, énfasis en el texto): si, por un lado, los individuos tiene derecho a explotar los terrenos naturales a través de su propio trabajo, por otro lado están legitimados a hacerlo solamente "at least where there is enough, and as good, left in common for others" (J. LOCKE, Second Treatise of Government, 1690, cap. V, par. 27).

18 C. DEL BÒ, I diritti sulle cose: Teorie della giustizia e validità dei titoli, cit., p. 87, énfasis en el texto.

19 V. en este sentido G.A. COHEN, Self-Ownership, World-Ownership, and Equality: Part I, en F. LUCASH (ed.), Justice and Equality Here and Now, Cornell University Press, Ithaca, 1986, pp. 108-135. 
la compatibilidad entre derechos, desde esta perspectiva, se presenta como exclusivamente definitorio o estipulativo, al depender en gran medida de la noción de derechos previamente aceptada.

\section{EL MINIMALISMO LIBERAL}

Al modelo nozickiano se puede conectar parcialmente una perspectiva bastante recurrente, dentro del panorama filosófico-político y filosófico-jurídico contemporáneo, que atribuye implícitamente a la sucesión histórica de las distintas categorías de derechos una distinción estructural, de carácter axiológico ${ }^{20}$, entre derechos "auto-ejecutivos" -los derechos civiles y políticos de la primera generación ${ }^{21}$ - y derechos a prestaciones positivas, de crédito hacia el Estado -los derechos sociales, económicos y culturales, así como los derechos colectivos, relacionados especialmente con las esferas de la bioética y de las tecnologías. Los primeros se caracterizarían por imponer meras abstenciones (evitar matar, torturar, imponer la censura, etc.), mientras que los segundos requerirían sobre todo, por no decir exclusivamente, el cumplimento de obligaciones positivas (erogaciones de recursos públicos, prestaciones en la esfera de la sanidad o de la instrucción, etc.). Dentro de este marco, los derechos de la tercera y de la cuarta generación (derechos colectivos y de solidaridad) son frecuentemente caracterizados como intereses cuya implementación exige una pluralidad de intervenciones, financiadas principalmente a través del recurso a la imposición y, por ello, potencialmente lesivos de los derechos de propiedad.

A partir del reconocimiento de la escasez natural de los recursos, los derechos sociales (aquellos derechos orientados a la neutralización de las desigualdades materiales presentes en la sociedad) ${ }^{22}$, así como los derechos "po-

20 Una reconstrucción parecida se puede encontrar, por ej., en M. IGNATIEFF, Human Rights as Politics and Idolatry, ed. by A. Gutmann, Princeton University Press, Princeton, Oxford, 2001; T. NAGEL, Mortal questions [1979], Cambridge University Press, Cambridge, 2013.

${ }_{21}$ V. por ej. C. FRIED, Right and Wrong, Harvard University Press, Cambridge (Massachusetts), 1978; M. CRANSTON, Human Rights, Real and Supposed, cit., p. 50 ("civil and political rights are not difficult to institute. For the most part, they require Governments, and other people generally, to leave a man alone [...] generally they can be secured by fairly simple legislation").

22 La función redistributiva de los derechos sociales se encuentra subrayada en particular en T.H. MARSHALL (Citizenship and social class [1950], Pluto Press, London, 1992), quien considera los derechos sociales como determinaciones cualitativamente nuevas de la condi- 
sitivos" en general (las pretensiones a una intervención positiva por parte de otros sujetos), son configurados como intereses cuyo cumplimiento corre el riesgo de conducir a ineficiencias económicas globales del sistema, colocando a los titulares en una condición de competitividad estructural para acceder a los bienes de la comunidad ${ }^{23}$. Estos derechos tienden a ser concebidos como meras "oportunidades condicionales" ${ }^{24}$, inevitablemente vinculadas a la discrecionalidad de las elecciones políticas parlamentarias y gubernamentales, y cuya implementación depende en gran parte de la disponibilidad de los recursos. Sobre la base de esta interpretación, la efectividad de los derechos socioeconómicos, culturales y de nueva generación depende en gran medida de las opciones sobre cómo redistribuir la riqueza nacional (por ejemplo, en el caso de la atención médica o de la instrucción pública), mientras que las libertades civiles y políticas pueden garantizarse independientemente de la coyuntura económica, sin menoscabar la arquitectura liberal de la sociedad.

En el plano jurisprudencial, podemos considerar sobre todo el trato específico reservado a los derechos fundamentales (en particular sociales) por el Tribunal de Justicia de la Unión Europea, aproximadamente en las últimas dos décadas: neto de algunas fluctuaciones ${ }^{25}$, el escenario más frecuente que

ción de ciudadanía y en tensión estructural con el mercado, dado que su inclusión implica la creación de un derecho a percibir una renta no proporcional al valor de mercado de quien lo reclama. A este respecto, véase también D. ZOLO, La strategia della cittadinanza, en Id. (a cura di), La cittadinanza. Appartenenza, identità, diritti, Laterza, Roma-Bari, 1994, pp. 33-34, quien advierte que el efectivo cumplimiento de los derechos sociales es incompatible con el ideal de la plena eficacia del mercado y, por tanto, sólo puede pasar por una profunda revisión de las estructuras de la economía capitalista. Por último, también S. RODOTÀ, Repertorio di fine secolo, Laterza, Roma-Bari, 1992, pp. 117, 120, alega que el reconocimiento de los derechos sociales representa una alternativa a la lógica de la autosuficiencia del mercado.

23 Para una postura de este tipo véanse en particular G. CORSO, "Diritti umani", Ragion pratica, núm. 7, 1996, pp. 59-66, que hace referencia a la idea de un "conflicto distributivo" entre los diferentes titulares de derechos sociales, utilizando también la metáfora de la sustracción como efecto de una acumulación continua de derechos, y A. PINTORE, I diritti della democrazia, Laterza, Roma-Bari, 2004, quien habla de un "juego de suma cero" en el que algunos derechos no son aplicados para dejar espacio a la protección de otros derechos.

24 Esta expression fue utilizada inicialmente por J.M. BARBALET en Citizenship: Rights, Struggle and Class Inequality, Open University Press, London, 1988.

25 Cabe señalar que, en algunas sentencias relativamente recientes, la Corte parece estar dispuesta a limitar las "libertades fundamentales" para proteger los "derechos fundamentales" reconocidos por las tradiciones constitucionales nacionales y por la CEDH, orientándose así hacia una colocación de los derechos y libertades en un terreno de igualdad. Véanse, en particular, del Tribunal de Justicia: 26 de junio de 1997, caso C-368/95, Familiapress; 12 de junio de 2003, caso C-112/00, Schmidberger; 14 de octubre de 2004, caso C-36/02, Omega. Finalmente,

ISSN: 1133-0937

DOI: https://doi.org/10.20318/dyl.2021.6109
DERECHOS Y LIBERTADES

Número 45, Época II, junio 2021, pp. 303-326 
resume su razonamiento jurídico al respecto es que los Estados miembros pueden aplicar los derechos reconocidos dentro de sus respectivos ordenamientos siempre y cuando no interfieran con la aplicación del derecho de la Unión y con el predominio uniforme de los derechos y libertades reconocidos y regulados por el mismo. En este sentido, son numerosas las decisiones del Tribunal de Justicia que demuestran, en general, que los derechos sociales sólo pueden ser reconocidos (ser elevados a la categoría de derechos fundamentales) si contribuyen a atribuir relevancia a las tradicionales libertades económicas de circulación de capitales, personas, bienes y servicios (las cuatro libertades del mercado común europeo $)^{26}$. No es exagerado afirmar que, en muchos casos, los derechos sociales (especialmente los derechos laborales y los derechos relacionados con los sistemas de seguridad social) están "funcionalizados" a las exigencias de competitividad del mercado común europeo, de la libre competencia y, en general, del desarrollo económico. De forma más o menos consciente según el caso, la distinción teórica entre las distintas generaciones de derechos acaba legitimando el trato desfavorable que los derechos de la tercera y cuarta generación suelen recibir.

Esta dicotomía, al estar desprovista de todo respaldo lógico, debe ser rechazada. En las consideraciones que siguen, destacaremos las principales aporías que socavan el modelo minimalista en sus dos variantes.

\section{LA IRRAZONABILIDAD DEL MINIMALISMO}

Dado que el minimalismo aspira a ser un proyecto filosófico-político de transformación social, su irracionalidad puede ser hallada exclusivamente en su impermeabilidad a los condicionamientos ejercidos por el derecho positivo. Sin embargo, cabe señalar que se aleja considerablemente de la realidad jurídica la pretensión, formulada por los teóricos minimalistas, de analizar las relaciones entre derechos a través de un análisis lingüístico y conceptual en el que el nivel semántico está completamente separado del contexto con-

en la sentencia Albany International BV contra Stichting Bedrijfspensioenfonds Textilindustrie (C$67 / 96,1999)$ la Corte ha declarado que el derecho a la negociación colectiva puede estar sustraído del condicionamiento ejercido por el derecho de competencia.

26 Entre las sentencias más representativas, se pueden considerar las decisiones relativas al derecho de huelga (sent. Viking: C-438/05, 2007), de lucha sindical (sent. Laval: C-341/05, 2007) y de salario mínimo (sent. Rüffert: C-346/06, 2008); véase también la sent. Comisión de las Comunidades Europeas contra Gran Ducado de Luxemburgo (C-319/06, 2008). 
creto de su implementación. En el campo de la interpretación jurídica y de la práctica jurisprudencial, la mayoría de las veces, la determinación de los límites de un derecho impone un trabajo de ponderación con otros derechos potencialmente conflictivos dentro de las circunstancias del caso específico.

Cristopher Wellman, en particular, al evaluar la propuesta nozickiana, señala que ni siquiera esta manera de configurar la relación entre derechos podría excluir la posibilidad de conflictos entre derechos negativos ${ }^{27}$. De manera similar, Bruno Celano afirma que incluso un conjunto minimalista de derechos liberales clásicos, para ser plausible, debería incluir una pluralidad de derechos susceptibles de colisionar entre sí (y por tanto de causar conflictos entre los deberes correlativos), así como el establecimiento de cláusulas abiertas, limitaciones y "términos éticos gruesos" 28.

La disciplina constitucional de los derechos muestra que la posibilidad de proteger cualquier derecho depende siempre de la activación de un conjunto de garantías jurisdiccionales. El recurso generalizado a técnicas ponderativas, tests de proporcionalidad y criterios de razonabilidad, así como las continuas reglamentaciones (especificaciones, determinaciones del contenido) que los tribunales constitucionales y los jueces comunes aplican a todo tipo de derechos, demuestran que incluso el ejercicio de los derechos de libertad puede ser gradual y estar vinculado a la disponibilidad coyuntural de los recursos materiales y jurídicos ${ }^{29}$.

Por tanto, incluso los derechos civiles y políticos tradicionales (libertad personal y libertad de movimiento, libertad de asociación y reunión, inviolabilidad del hogar, propiedad privada, etc.), generalmente asociados a las fun-

27 "Rejecting positive rights cannot solve the problem of conflicting rights" (C. WELLMAN, On Conflicts Between Rights, The Proliferation of Rights. Moral Progress or Empty Rhetoric?, Westview, Boulder, 1999, p. 273).

${ }_{28}$ "[A]nche un Bill of Rights che si limiti a sancire i diritti di libertà (diritti della prima, o della primissima, generazione), riducendo all'essenziale il catalogo dei diritti costituzionalmente sanciti, purché ragionevole e sensato, genera la possibilità di conflitti, tensioni, fra i diritti, e fra questi ultimi e ulteriori fini, scopi, obiettivi, valori sociali e politici; e genera, in tal modo, l'indeterminatezza e l'incertezza tipiche delle costituzioni contemporanee" (B. CELANO, I diritti nello Stato costituzionale, Il Mulino, Bologna, 2013, p. 96).

29 Sobre la coexistencia de las dimensiones positiva y negativa de los derechos, véanse en particular J. WALDRON, Rights in Conflict, cit., p. 214 ("one and the same right may generate both negative and positive duties"); L. FERRAJOLI, Principia iuris. Teoria del diritto e della democrazia. Vol. 1. Teoria del diritto, Laterza, Roma-Bari, 2007, pp. 325, 327; S. HOLMES, C.R. SUNSTEIN, The Cost of Rights: Why Liberty Depends on Taxes, Norton \& Company, New York, London, 1999. 
ciones típicas del Estado de derecho liberal, requieren, para volverse efectivos, intervenciones positivas como el establecimiento de sistemas burocráticos, administrativos y judiciales, el ejercicio del poder policial y otras funciones. Se puede pensar, por ejemplo, en el caso del derecho a la participación democrática, que en la práctica requieren mucho más que la mera abstención por parte del Estado, ya que siempre son necesarias algunas estructuras políticas que brinden un espacio adecuado para la participación popular ${ }^{30}$.

Al mismo tiempo, ni siquiera los derechos socio-económicos, culturales y de la cuarta generación pueden ser vistos simplemente como el resultado de obligaciones positivas: aun cuando los titulares ya hayan tenido acceso al bien que constituye el objeto del derecho en cuestión, el poder estatal seguirá teniendo que abstenerse de realizar aquellas conductas que puedan dañarlo (por ejemplo, en el caso del libertad de sindicalización o derecho de huelga), y utilizar las medidas de control adecuadas para prevenir y sancionar las violaciones. Al respecto, J.A. Cruz Parcero correctamente señala:

cuando hablamos del derecho a la educación pensamos inmediatamente en la obligación positiva del Estado de impartir educación, construir escuelas, pagar a los maestros, dar becas, etc.; pero, a su vez, el Estado tiene obligaciones pasivas de no empeorar la educación, de no expulsar a los niños de las escuelas, de no negar el acceso a la educación (en ciertas circunstancias), de no cobrar cuotas de inscripción o colegiatura cuando la educación es gratuita, etc. ${ }^{31}$.

Además, no se puede pasar por alto que los mismos fondos públicos utilizados para financiar la protección de los derechos pueden constituir, en una perspectiva orientada hacia el futuro, una inversión productiva para incrementar el bienestar colectivo. En este sentido, retomando parcialmente una tesis de Amartya Sen -quien destaca la conexión entre el desarrollo económico, industrial y tecnológico de Japón y China a principios del siglo XX y las

30 Al respecto, observa Jeremy Waldron: “[r]eflection on the rights of the citizen also undermines the claim that first generation rights call only for inaction by the state, rather that collective intervention. In fact, rights to democratic participation require much more than mere omissions by the state. They require officials to approach their task in a certain spirit, and they require the establishment of political structures to provide a place for popular participation and to implement people's wishes, expressed by voting and other forms of pressure" (J. WALDRON, Can Communal Goods be Human Rights? [1987], en Liberal Rights. Collected papers 1981-91, cit., p. 343).

31 J.A. CRUZ PARCERO, El lenguaje de los derechos. Ensayo para una teoría estructural de los derechos, Trotta, Madrid, 2007, p. 76. 
inversiones en educación e investigación científica realizadas, en el período anterior- Luigi Ferrajoli sostiene acertadamente que "si es verdad que los derechos fundamentales tienen un costo, también es cierto que cuestan mucho más sus vulneraciones o sus incumplimientos" ${ }^{32}$. Especialmente en las democracias contemporáneas, caracterizadas por un estrecho vínculo entre el progreso económico, cultural y tecnológico, el reconocimiento y la protección de derechos esenciales como el acceso a la alimentación, a la seguridad social, a la salud o a la educación constituyen la base de la supervivencia individual, pero también del desarrollo socio-económico de la comunidad en su conjunto.

\subsection{Algunas observaciones sobre la interest theory y el enfoque dinámico}

4.1.1. El reconocimiento de la paritariedad de los derechos: algunas etapas esenciales

Dejando de lado el análisis de la teoría de Nozick, se puede observar que, en términos generales, la propuesta minimalista tiende a apoyarse en la identificación entre derechos y obligaciones jurídicas. Dicho de manera simple, con base en este enfoque, se tienden a considerar como dignos de protección solo aquellos bienes (auténticos, genuinos, gratuitos, etc.) cuyas garantías jurídicas ya han sido establecidas (o que puedan serlo fácilmente).

Ahora bien, una posible solución para superar algunos de los principales aspectos problemáticos implícitos en este enfoque es ofrecido por la interest theory contemporánea combinada con una perspectiva dinámica ${ }^{33}$ : la idea de que un mismo derecho constitucional puede originar más derechos, reivindicaciones y obligaciones de terceros, según un conjunto de direcciones no determinables a priori $^{34}$.

32 V. L. FERRAJOLI, Principia iuris. Teoria del diritto e della democrazia, vol. 2, cit., pp. 67-71, traducción mía; A. SEN, Resources, Values and Development, Harvard University Press, Harvard, 1984.

33 El hecho de que el enfoque dinámico, la mayoría de las veces, parezca estar estrechamente vinculado a diferentes formas de interest theories puede verse como históricamente contingente y desprovisto de necesidad lógica. En este punto ver L. SUMNER, The Moral Foundation of Rights, Oxford Clarendon Press, Oxford, 1987, pp. 39 ss., 51-53, 96 ss., quien elabora una versión de la choice theory (el enfoque que justifica la atribución de un derecho como una forma de proteger una elección) que acepta las tesis esenciales de la concepción dinámica.

34 La concepción dinámica -observa Celano- "vede un diritto soggettivo come il nucleo germinale di (come una ragione atta a giustificare l'attribuzione o il riconoscimento di) posi- 
Aunque existan diferencias notables al respecto, siguiendo a M.H. Kramer, podemos afirmar que todas las reconstrucciones que pueden ser incluidas bajo la denominación de 'interest theory' comparten dos principios básicos: “(1) [n] ecesario aunque insuficiente para que un sujeto $X$ disponga efectivamente de un derecho es que este mismo derecho, si efectivo, preserve uno o más de sus intereses. (2) El hecho de que $\mathrm{X}$ sea competente y autorizado para exigir o renunciar al ejercicio del derecho no es ni suficiente ni necesario para que $X$ posea tal derecho" ${ }^{35}$. Cabe matizar que, dentro de este esquema, la condición decisiva es esta última: dado que la competencia presupone la capacidad de elegir, si una persona no es moral o legalmente competente, ni siquiera puede ser autorizada moral o jurídicamente a tomar una elección. Esta es la razón principal por la que, para los partidarios de la choice theory, los niños o las personas que no están en condiciones de comprender y expresar una voluntad racional, ya que carecen de la capacidad de controlar y determinar sus elecciones, no se pueden considerar auténticos titulares de derechos. En otras palabras, los derechos son reivindicaciones acompañadas por un poder: sin poder puede haber una reivindicación, pero no existe ningún derecho. Por otro lado, según los defensores de la teoría del interés, la posesión de un derecho no depende de la posesión de poderes jurídicos sino únicamente del hecho de que este derecho protege los intereses de las personas.

La elaboración más completa de un enfoque de este tipo conjugado con una concepción dinámica tiene sus raíces, en la década de 1970, en algunas

zioni normative determinate, o insiemi determinati di posizioni siffatte (come la ratio che spiega, giustificandola, l'attribuzione o il riconoscimento di posizioni normative determinate)" (B. CELANO, "I diritti nella Jurisprudence anglosassone contemporanea", en Analisi e diritto 2001. Ricerche di giurisprudenza analitica, a cura di P. Comanducci, R. Guastini, Giappichelli, Torino, 2001, p. 6.). Enfoques dinámicos pueden encontrarse en: J. FEINBERG, “The Nature and Value of Rights", Journal of Value Inquiry, núm. 4, 1970, pp. 243-257; N. MACCORMICK, “Children's Rights: a Test-Case for Theories of Rights", Archiv für Rechts - und Sozialphilosophie, núm. 62, 1976, pp. 305-316; ID., Rights in Legislation, en P. HACKER, J. RAZ (ed. by), Law, Morality and Society, Oxford Clarendon Press, Oxford, 1977, pp. 188, 201; J. RAZ, Legal Rights [1984], reed. in Ethics in the Public Domain, Oxford Clarendon Press, Oxford, 1994; Id., The Nature of Rights [1984], reed. en Id., The Morality of Freedom, Oxford Clarendon Press, Oxford, 1986, pp. 165192; L. SUMNER, The Moral Foundation of Rights, cit., pp. 51-52; J. WALDRON, The Right to Private Property, Oxford Clarendon Press, Oxford, 1988, pp. 79-87; J. WALDRON, Rights in Conflict, cit., espec. pp. 212-214; D. LYONS, Rights, Claimants and Beneficiaries [1969], en Id., Rights, Welfare, and Mill's Moral Theory, Oxford University Press, New York, 1994.

35 M.H. KRAMER, Rights without Trimmings, en Id., N.E. SIMMONDS, H. STEINER (eds.), A Debate over Rights. Philosophical Enquiries, Oxford University Press, Oxford, 1998, p. 62 .

DERECHOS Y LIBERTADES

ISSN: 1133-0937

Número 45, Época II, junio 2021, pp. 303-326

DOI: https://doi.org/10.20318/dyl.2021.6109 
obras de Neil MacCormick (y más tarde retomadas y desarrolladas especialmente por Joseph Raz, Jeremy Waldron y Andrei Marmor), en las que el autor mueve una serie de objeciones a la perspectiva hartiana, optando por una visión dinámica de los derechos, entendidos como "situaciones favorables" de carácter argumentativo o justificativo ${ }^{36}$.

Sin embargo, antes de ilustrar esta posición, es necesario dar un paso atrás. Según H.L.A. Hart, en definitiva, el significado de los términos referidos al vocabulario de los derechos tiene que ser hallado en ese único elemento común a las diferentes nociones (o a la mayoría de ellas) que lo integran. Analizando la particular versión de la "benefit theory" presentada por Jeremy Bentham ${ }^{37}$, Hart $^{38}$ afirma que ser beneficiario del cumplimiento de una obligación jurídica no constituye una condición ni necesaria ni suficiente para ser titular del derecho correlativo a esta obligación. Por el contrario, es necesario y suficiente que el titular disponga del poder de control sobre la obligación correlativa a aquel derecho.

La perspectiva de la choice o will theory ${ }^{39}$-afirma el autor- "centrada en la noción de elección individual jurídicamente protegida" ${ }^{40}$, no puede ser

36 V. N. MAcCORMICK, "Children's Rights: A Test Case for Theories of Rights”, cit.; Id., Rights in Legislation, cit. Algunas anticipaciones de las tesis formuladas por MacCormick pueden encontrarse en D. LYONS, Rights, Claimants and Beneficiaries, cit.

37 El núcleo de la 'benefit theory' de los derechos, en la versión desarrollada por Jeremy Bentham, se puede resumir en los siguientes términos: "this identification of a right-holder by reference to the person or persons intended to benefit by the performance of an obligation" (H.L.A. HART, Legal Rights [1973], en Essays on Bentham. Studies in Jurisprudence and Political Theory, Oxford Clarendon Press, Oxford, 1982, p. 169).

38 Véase en particular H.L.A. HART, Are There Any Natural Rights? [1955], en J. WALDRON (ed.), Theories of Rights, Oxford University Press, Oxford, 1984, p. 35; ID., Legal Rights, cit., pp. 162-193. Sobre la posición tomada por Hart a partir de 1955, v. en particular J. WALDRON, Can Communal Goods be Human Rights? [1987], cit., pp. 366-367.

39 Aunque incluso en este caso existan varias diferencias, según M.H. KRAMER, los defensores de la choice theory comparten tres principios: “(1a) [s] ufficient and necessary for X's holding of a right is that $X$ is competent and authorized to demand or waive the enforcement of the right. (2a) $X^{\prime}$ s holding of a right does not necessarily involve the protection of one of more of $X^{\prime}$ s interests. (3a) A right's potential to protect one or more of $X^{\prime}$ 's interests is not sufficient per se for $X^{\prime}$ 's actual possession of that right" (M.H. KRAMER, Rights without Trimmings, cit., p. 62). Versiones emblemáticas de la choice theory pueden ser encontradas en H.L.A. HART, Legal Rights, cit.; N.E. SIMMONDS, Rights at the Cutting Edge, en M.H. KRAMER, N.E. SIMMONDS, H. STEINER (ed.), A Debate over Rights, cit.; H. STEINER, Working Rights, en M.H. KRAMER, N.E. SIMMONDS, H. STEINER (ed.), A Debate over Rights, cit., pp. 233-301; C. WELLMAN, An Approach to Rights: Studies in the Philosophy of Law and Morals, Kluwer, Dordrecht, 1997.

$40 \quad$ H.L.A. HART, Legal Rights, cit., p. 189.

ISSN: 1133-0937

DOI: https://doi.org/10.20318/dyl.2021.6109
DERECHOS Y LIBERTADES

Número 45, Época II, junio 2021, pp. 303-326 
considerada exhaustiva con respecto a la noción de derecho subjetivo ${ }^{41}$, ya que no puede proporcionar explicaciones adecuadas para todos aquellos casos en los que parece apropiada un utilización del lenguaje de los derechos referido a las necesidades básicas, entendidas como bienes esenciales para la preservación de la dignidad de los individuos ${ }^{42}$. En virtud de sus características, este planteamiento no puede ser considerado como la única explicación posible para los derechos subjetivos ${ }^{43}$ : en algunos casos, es posible argumentar que el núcleo de la noción de derecho subjetivo, más que en la posibilidad de elección por parte de los titulares de derechos, tiene que ser hallado en la idea de necesidades básicas. Esto se da principalmente en dos ámbitos: el de los sistemas constitucionales rígidos -en los que los derechos constituyen al mismo tiempo límites formales y sustanciales para el legislador- y en el ámbito de la evaluación moral, es decir, en un contexto radicalmente diferente respecto al vocabulario "estándar" de los derechos.

El propio Hart, en otras palabras, a pesar de poner énfasis en la relevancia de la elección individual en su definición de la noción de derecho subjetivo, reconoce que las concepciones voluntaristas no pueden dar cuenta de aquellas expectativas cuyo cumplimiento no puede delegarse a un acto potestativo del titular (la facultad de determinar el comportamiento de otro sujeto), sino que depende de la iniciativa de otro sujeto. Tales teorías no parecen capaces de captar la noción de derecho subjetivo que, a partir de unas necesidades fundamentales, sería ajena al lenguaje técnico-jurídico habitualmente empleado por jueces, abogados, juristas, perteneciendo más bien a "una forma peculiar de crítica moral del derecho" ${ }^{44}$. La validez de la teoría

41 Sobre los límites que el propio Hart reconoce en la teoría de la elección, ver J. FINNIS, Natural Law and Natural Rights, Oxford Clarendon Press, Oxford, 1980, pp. 204-205.

42 Siguiendo la reconstrucción de LUIGI FERRAJOLI (Diritti fondamentali: un dibattito teorico, cit., pp. 298-299), se puede suponer que fundar derechos equivale a plantearse el problema de determinar su sustrato axiológico y a proporcionar las razones por las que deben ser reconocidos dentro del ordenamiento jurídico. En un nivel general de ética normativa, en una perspectiva "filosófico-política" o de "teoría de la justicia", fundar los derechos humanos equivale a explicitar los argumentos a favor de su existencia y su legitimidad. De acuerdo con esta línea de razonamiento, podemos identificar el fundamento de la dignidad humana en el valor de la existencia del individuo como titular de derechos y deberes morales (véase en este sentido J. HABERMAS, Die Zukunft der menschlichen Natur. Auf dem Weg zu einer liberalen Eugenik?, Suhrkamp, Frankfurt am Main, 2001).

43 V. H.L.A. HART, Legal Rights, cit., p. 189.

44 Ibid., pp. 192-193. En relación con el caso específico de los welfare rights, Hart sostiene que estos derechos pueden ser reclamados a través de una perspectiva voluntarista en al menos dos contextos: cuando la aplicación de la prestación positiva que es objeto del derecho 
de la elección, por tanto, se limitaría a la justificación de algunos derechos jurídicos: de aquellos casos en los que el titular de un derecho está en condiciones de ejercer un poder sobre el destinatario del deber. Por otro lado, Hart reconoce que este enfoque resulta inadecuado para explicar ciertas cuestiones relacionadas con la protección del bienestar y el desarrollo humanos: en estos casos, la noción de beneficio o interés nos permite explicar en qué manera los individuos son tutelados, a través de "derechos-inmunidad" (a los que corresponden "discapacidades" para el legislador), frente a los abusos del poder estatal organizado ${ }^{45}$.

Basándose en estas intuiciones, será sobre todo Neil MacCormick quien deconstruirá la (que en su momento constituía la) teoría estándar, introduciendo una serie de contraejemplos para mostrar que algunos titulares de derechos pueden carecer de cualquier poder sobre las obligaciones correlativas de otros sujetos. El ejemplo paradigmático que hace el autor, como es sabido, es el de los derechos de los niños: es sensatamente posible argumentar que todo niño tiene derecho a recibir una instrucción, sin saber todavía con precisión quién debe tener la obligación correspondiente y el poder de proporcionarla $^{46}$. Un segundo ejemplo se refiere a otros titulares de derechos indisponibles, en particular en el ámbito de las relaciones laborales y el poder de negociación, quienes tienen plena capacidad de actuación pero que, por diferentes motivos, no hacen uso (o no pueden hacerlo) de ningún poder para elegir entre el cumplimiento o la no obligación correlativa ${ }^{47}$. Según

esté condicionada a una petición expresa por parte del titular del derecho, y cuando la ley prevea ciertos mecanismos de enforcement en caso de que no se preste el servicio. Sin embargo, no se puede reconducir a un enfoque voluntarista el caso de los derechos sociales en relación con los cuales el titular del deber correspondiente no es identificable o, por otros motivos, la prestación no resulta exigible (v. ibid., pp. 185-186).

45 Ibid., pp. 168-9. Simplificando: un individuo $X$ tiene una inmunidad frente a un individuo $Y$ si este último no tiene el poder de (no está en condiciones de) modificar la posición jurídica de $X$. Señalan cómo es posible dar cuenta de los derechos de inmunidad sobre la base de un enfoque voluntarista: N. MACCORMICK, Rights in Legislation, cit., pp. 194-5; M. BAYLES, Hart's Legal Philosophy. An Examination, Kluwer, Dordrecht, 1992, p. 147. En una primera fase de su reflexión -hay que precisar- Hart se inclina a explicar la noción de inmunidad desde la perspectiva de la teoría de la elección (v. H.L.A. HART, Definition and Theory in Jurisprudence [1953], en Essays in Jurisprudence and Philosophy, Oxford Clarendon Press, Oxford, 1983, p. 35 n.).

46 N. MACCORMICK, Children's Rights: A Test Case for Theories of Rights, cit., pp. 305, 313. Sobre este tema, véase el análisis I. FANLO CORTÉS, I bambini e i diritti. Una relazione problematica, Giappichelli, Torino, 2008. Por una reconstrucción del debate Hart-MacCormick, v. B. CELANO, I diritti nello Stato costituzionale, cit., espec. pp. 54-65.

47 N. MAcCORMICK, Rights in Legislation, cit., pp. 196-197.

ISSN: 1133-0937

DOI: https://doi.org/10.20318/dyl.2021.6109
DERECHOS Y LIBERTADES

Número 45, Época II, junio 2021, pp. 303-326 
MacCormick, por lo tanto, las disposiciones jurídicas no constituyen en sí mismas un derecho, pero son esenciales para llevarlo a la práctica, "al ampliar los derechos de los niños" ${ }^{48}$. Cuando se afirma, por ejemplo, que un niño tiene derecho a ser alimentado, eso no significa simplemente que otra persona tiene el deber de alimentarlo, sino que la satisfacción de su interés es un fin en sí mismo y no un medio para lograr otros fines ${ }^{49}$.

Ahora bien, uno de los principales aspectos críticos de esta orientación teórica es que a la existencia de un derecho moral (y del deber moral relacionado) no siempre corresponde un deber jurídico: en otras palabras, el riesgo inherente a este enfoque sería el de desconocer la relevancia de la adopción autoritativa de decisiones vinculantes. Al respecto, MacCormick mantiene, sin embargo, que pueden existir derechos jurídicos (no solo derechos morales ${ }^{50}$ ) no correlativos y lógicamente antecedentes respecto a la determinación de deberes jurídicos: la función de los derechos no es reducible en modo alguno a la de meros epifenómenos de deberes u otras posiciones jurídicas. Una posible respuesta a este problema se encuentra en la distinción, elaborada por Ferrajoli, entre garantías primarias (obligaciones positivas o negativas, asumidas por particulares y/o autoridades públicas, relacionadas con

48 Id., Children's Rights: A Test Case for Theories of Rights, cit., p. 308. El propio MacCormick, sin embargo, no desconoce la relevancia del papel de la elección individual en el ejercicio de los derechos subjetivos (" $\mathrm{i}$ ] $\mathrm{t}$ is certainly true that apart from such cases as those of children or the mentally incapacitated, the holder of a legal right is normally permitted and empowered in law to choose whether or not on any given occasion he should avail himself of his right"; N. MAcCORMICK, Children's Rights, cit., p. 314).

49 V. Id., Children's Rights, cit., p. 159.

50 En términos generales, con la expresión 'derechos morales' podemos referirnos a aquellos derechos ideales que surgen de la dimensión ética de la existencia del individuo, anterior a las instituciones sociales, que para ser afirmados o reivindicados no requieren de ningún tipo de reconocimiento jurídico o institucional. Particularmente esclarecedor, al respecto, es la reflexión de Carlos S. Nino quien, reformulando una definición de MAcCORMICK (Legal Right and Social Democracy. Essays in Legal and Political Philosophy, Oxford Clarendon Press, Oxford, 1982, p. 160), caracteriza a los derechos morales en los siguientes términos: «[s]e adscribe a alguien el derecho de acceder a una situación S (que puede ser la posibilidad de realizar cierta acción o la de disponer de determinados recursos o la de verse librado de ciertas contingencias) cuando el individuo en cuestión pertenece a una clase $\mathrm{C}$ y se presupone que $S$ implica normalmente para cada miembro de $C$ un bien de tal importancia que debe facilitarse su acceso a $S$ y es moralmente erróneo impedir tal acceso, cualesquiera que sean las consecuencias de tal acceso respecto de valores agregativos" (C.S. NINO, Ética y derechos humanos Un ensayo de fundamentación [1984], Astrea, Buenos Aires, 1989, p. 40). De acuerdo con esta perspectiva, de hecho, el único requisito para atribuir un derecho moral a un sujeto está constituido por la clase C (la persona moral). 
un derecho) y garantías secundarias (la obligación de los jueces de sancionar con la anulación o con la condena los hechos inválidos o ilegales en los que ocurran las violaciones a las garantías primarias ${ }^{51}$ ). Según el autor, aunque los derechos consisten en "expectativas negativas o positivas a las que corresponden obligaciones (de provisiones públicas) o prohibiciones (de lesiones)" ${ }^{52}$, y por lo tanto las obligaciones y prohibiciones que les corresponden, así como las obligaciones conexas de reparar o sancionar judicialmente eventuales lesiones de derechos, "derivan lógicamente por el propio estatuto normativo de los derechos", en la práctica suele ocurrir que no sólo se infringen estas obligaciones y/o prohibiciones, sino que "ni siquiera se establece por ley" ${ }^{33}$. Sin embargo, la falta de mecanismos de protección a nivel infraconstitucional, y en particular en lo que respecta al aparato de garantías jurisdiccionales, no anula la existencia de un derecho, sino que configura una laguna dentro del ordenamiento jurídico ${ }^{54}$. En este caso, la responsabilidad de colmar la laguna recae sobre el legislador o sobre el juez-intérprete: en el caso de que la relativa meta-obligación quede incumplida, no obstante, persistirá el problema de identificar las garantías secundarias del derecho en cuestión.

\subsubsection{Argumentos críticos contra el minimalismo}

De acuerdo con los partidarios de la interest theory, la mayor eficacia de este enfoque depende principalmente de su capacidad de justificar el reconocimiento, al menos a nivel moral prepositivo, de los derechos sociales ${ }^{55} \mathrm{y}$

51 V. L. FERRAJOLI, Principia iuris. Teoria del diritto e della democrazia, cit., vol. I, pp. 196-198, 668-701; Id., La logica del diritto. Dieci aporie nell'opera di Hans Kelsen, Laterza, RomaBari, 2016, espec. cap. II, III.

52 Id., Diritti fondamentali. Un dibattito teorico, cit., p. 11, traducción mía.

53 Id., "Diritti fondamentali", Teoria politica, núm. 14, vol. 2, 1998, p. 11, n. 2.

54 V. Id., La democrazia attraverso i diritti. Il costituzionalismo garantista come modello teorico e come progetto politico, Laterza, Roma-Bari, 2013, pp. 53-54

55 Como destaca Alessandra Facchi, sin embargo, "sostenere la centralità della scelta e della volontà individuale non significa escludere la rilevanza dei diritti economici-sociali" (A. FACCHI, "Diritti fantasma? Considerazioni attuali sulla proliferazione dei soggetti", Ragion pratica, núm. 31, 2008, p. 325). En este sentido, no hay impedimento, en principio, para recurrir a una justificación de los derechos sociales que haga referencia a una perspectiva de will o choice theory. Sobre la compatibilidad entre interest theory y (la protección de los) derechos sociales, J. WALDRON (ed.), Theories of Rights, cit., pp. 11-12; Id., Liberal Rights: Two Sides of the Coin, cit., pp. 16-17. Por una posición que coloca el fundamento de los derechos sociales en el respeto de la autonomía individual, v. C. FABRE, Social Rights Under the Constitution. Government and the Decent Life, Oxford University Press, New York, 2004.

ISSN: 1133-0937

DOI: https://doi.org/10.20318/dyl.2021.6109
DERECHOS Y LIBERTADES

Número 45, Época II, junio 2021, pp. 303-326 
de los derechos atribuidos a sujetos que no son capaces de expresar su voluntad (los niños, de algunas personas con discapacidad, de generaciones futuras, etc.). Sobre la base de esta perspectiva, es posible reconocer la existencia de un derecho cuando un determinado interés (su elemento justificativo) se considera suficientemente importante para atribuir a un sujeto titular una serie de posiciones subjetivas favorables (libertades, pretensiones, poderes e inmunidades), e imponer a otro sujeto (no necesariamente a un individuo específico) las posiciones correlativas necesarias para protegerlo. En este sentido, se hace posible incluir en el catálogo de los derechos aquellos casos cuyos titulares no están en condiciones de realizar elecciones libres y conscientes ${ }^{56}$.

Además, este planteamiento también permitiría incluir una gama de opciones más amplias entre las posibles justificaciones de los derechos, aceptando diferentes valores (principios, bienes, necesidades, etc.) como justificaciones del reconocimiento de derechos, que incluyen también la protección de la libertad individual. Una reconstrucción doctrinal que identifica el núcleo generativo de los derechos en la tutela de un interés individual o colectivo puede aceptar diferentes valores como elementos justificativos del reconocimiento de derechos, al resultar más abierto frente a los cambios del marco social de referencia. Este enfoque, que reconoce, como podría decirse, un "fundamento suficiente" $57 \mathrm{o}$ "distintos fundamentos posibles" 58 de los derechos, parece más compatible con un contexto de pluralismo ideológico como el que caracteriza al constitucionalismo contemporáneo.

Los derechos, en este sentido, se conciben como exigencias ético-jurídicas que constituyen el núcleo de una pluralidad de posiciones y deberes subjetivos ("grounds of duties") 59 ; posiciones complejas que incluyen tanto características de intervención "positiva" como de abstención "negativa", y de las que surgen varias "oleadas" ("waves") de posiciones subjetivas específicas no predeterminables. Observa al respecto Celano:

56 V. por ej. M.H. KRAMER, Rights without Trimmings, cit., p. 64.

57 V. C. PERELMAN, Peut-on fonder les droits de l'homme?, en Droit, morale et philosophie, Librairie générale de droit et de jurisprudence, Paris, 1968, pp. 57-64.

58 V. N. BOBBIO, "Sul fondamento dei diritti dell'uomo" [1965], en ID, L'età dei diritti, Einaudi, Torino, 1992.

59 "The essential point is, however, that rights are grounded in the interests of the potential right holder, and that duties are grounded in rights" (A. MARMOR, On the limits of rights, cit., pp. 3-4). 
[p]ara quien adopte la choice theory, es difícil encontrar razones en apoyo la conclusión de que necesidades e intereses humanos básicos constituyen, como tales, la base de los derechos. Si, por el contrario, se adopta la interest theory -es decir, si se asume que existe un derecho siempre que haya un interés (un bien) que tiene que ser protegido, o cumplido (un interés suficientemente importante como para justificar la imposición de obligaciones a otros sujetos)- la posibilidad de calificar lo que constituye el objeto de necesidades o intereses humanos fundamentales como, de por sí, objeto de otros tantos derechos no presenta ninguna dificultad conceptual. En esta perspectiva, los derechos de libertad se configuran como una clase particular de derechos, iguales y coordinados con respecto a los demás. De la misma manera que la vida y las condiciones materiales necesarias para una existencia digna, incluso la libertad, o algunas libertades, son bienes que deben garantizarse a los individuos (son intereses que deben protegerse) ${ }^{60}$.

La interest theory, combinada con una perspectiva dinámica, tiende a atribuir la misma dignidad teórica a las diferentes generaciones de los derechos, incluso cuando ciertos intereses aún no hayan sido aceptados dentro de un ordenamiento jurídico positivo y su reivindicación tiene sentido solo desde el punto de vista de la crítica moral del derecho. En este sentido, la validez del planteamiento en cuestión parece encontrar su confirmación en el funcionamiento efectivo de las disposiciones que atribuyen derechos fundamentales: además de los perfiles de abstención negativa, siempre se requiere, para los fines de su efectivización, un trabajo complejo de determinación (especificación, concreción, delimitación) de su contenido mediante la creación de apropiados institutos, normas y reglamentos, y de la predisposición de los necesarios recursos económicos y financieros ${ }^{61}$.

De hecho, esta reconstrucción del significado de los derechos puede aportar distintos elementos teóricos para rechazar aquella lógica economicista ${ }^{62}$ que asigna una importancia central al "principio de necesidad" impuesto

60 B. CELANO, "I diritti nella Jurisprudence anglosassone contemporanea. Da Hart a Raz", cit., pp. 51-52, traducción mía.

61 En este sentido, véanse sobre todo R. BIN, “Diritti e fraintendimenti”, cit., pp. 1525; E. DICIOTTI, Il mercato delle libertà: l'incompatibilità tra proprietà privata e diritti, Il Mulino, Bologna, 2006, pp. 86 ss.; S. HOLMES, C.R. SUNSTEIN, The Cost of Rights: Why Liberty Depends on Taxes, cit.; R.A. POSNER, The Twilight of Human Rights Law, Oxford University Press, Oxford, 2014; P. HÄBERLE, Los derechos fundamentales en el Estado prestacional, Palestra, Lima, 2019.

62 Por una crítica al proyecto de extender la metodología económica al análisis de los procesos políticos y a la fundamentación de los derechos humanos, véase en particular

ISSN: 1133-0937

DOI: https://doi.org/10.20318/dyl.2021.6109
DERECHOS Y LIBERTADES

Número 45, Época II, junio 2021, pp. 303-326 
por los mercados, y por las instituciones monetarias y bancarias: una especie de planteamiento jurisprudencial que asigna a los derechos sociales un papel de subordinación instrumental en relación con los objetivos económicos. Este tipo de propuesta acerca del fundamento teórico de los derechos puede contribuir a resaltar la inconsistencia del modelo de razonamiento que subyace en varias decisiones de los tribunales constitucionales, al tiempo que sienta las bases para una concepción alternativa de los mismos, dentro de una visión orientada a repristinar la supremacía de la dimensión ética de los derechos sobre otras necesidades y exigencias (a partir de los procesos socioeconómicos).

J. RAWLS, Theory of Justice: Revised Edition [1971], Harvard University Press, Cambridge, Massachusetts, 1999, p. 317. 